\title{
Lipase Hypersecretion Syndrome
}

National Cancer Institute

\section{Source}

National Cancer Institute. Lipase Hypersecretion Syndrome. NCI Thesaurus. Code C60825.

A rare disorder resulting from neoplastic exocrine excess. It is typically seen in 10-15\% of patients with pancreatic acinar cell carcinoma. Clinical signs include subcutaneous fat necrosis, polyarthralgia and osteolytic lesions. Clinical course may proceed to nonbacterial thrombotic endocarditis. Clinical prognosis is poor due to its usual onset with advanced disease. 